should grow, due to ${ }^{3} \mathrm{He}$ production from hydrogen by low-mass stars ${ }^{10}$. The measurement by Gloeckler and Geiss is inconsistent with this prediction because the sum of the local deuterium and ${ }^{3} \mathrm{He}$ abundances is essentially equal to that inferred for the Solar System 4.5 billion years ago. At the moment, any argument for or against a particular value of the primeval deuterium abundance based upon the chemical evolution of ${ }^{3} \mathrm{He}$ has little weight.

There are also pieces missing from the ${ }^{7} \mathrm{Li}$ story. Its abundance in the atmos- pheres of the oldest stars in the halo of the Galaxy is $(1.4 \pm 0.3) \times 10^{-10}$. If this is the primordial value, it favours a low baryon density. However, some stellar models indicate that these stars could have reduced their initial ${ }^{7} \mathrm{Li}$ abundance by a factor as large as two, which would be consistent with a high baryon density.

It is a good bet that within a few years the primeval deuterium abundance will be determined unambiguously, and with it the value of the baryon density. An important check may come a few years later from detailed mapping of the anisotropy of the cosmic background radiation, which provides an independent way of determining the baryon density to similar precision ${ }^{11}$. Once the baryon density is known, nuclear physics in the early Universe can teach us about the 'chemistry' of ${ }^{3} \mathrm{He}$ and ${ }^{7} \mathrm{Li}$ in the contemporary Universe.

David N. Schramm and Michael S. Turner are in the Department of Astronomy and Astrophysics, and the Department of Physics, University of Chicago, Chicago, Illinois 60637-1433, USA.

\section{Blindness and the $X$}

\section{Sean E. Egan and Roderick R. Mclnnes}

PositionAL cloners, explorers of undefined but promising genetic territory, are as vulnerable to confounding data as were any of their nineteenth-century counterparts in mapping the head of the Nile. But the longest lasting chromosomal walk to date has now ended happily, 12 years after Bhattacharya and his colleagues ${ }^{1}$ found a locus for $\mathrm{X}$-linked retinitis pigmentosa (xlRP) on the short arm of the $\mathrm{X}$ chromosome.

As reported in the May issue of Nature Genetics $^{2}$, two previously competing groups joined in an admirable collaboration to find an RP gene on Xp at the locus (RP3) associated with more cases of RP than any other known, up to around $20 \%$ in some populations. Discovery of the new gene, named $R P G R$ (for retinitis pigmen- tosa GTPase regulator) has important implications for retinal biology, and for patients with the disease and their families.

Retinitis pigmentosa is a relatively common genetic disorder which affects 1 in 4,000 people and is caused by the premature death of photoreceptors. The result is progressive night blindness, contraction of the visual field and, finally, complete loss of sight. X-linked RP is one of the more severe forms; it usually becomes manifest within the first two decades of life, and progresses to blindness within 10-20 years ${ }^{3}$.

What made the search for $R P 3$ so tortuous? First, the genetic analysis of xIRP was plagued by unexpected locus heterogeneity. The initial impression of a single xIRP locus ${ }^{1}$ has been expanded to include four putative loci $(R P 2, R P 3, R P 6$ and $R P 15)$ between $\mathrm{Xp} 11.22$ and $\mathrm{Xp} 22.13$ (refs 4-8), although the genetic evidence is undeniable only for the existence of $R P 2$ (in $\mathrm{Xp} 11.3-11.23$ ) and $R P 3$ (in $\mathrm{Xp} 21.1)^{5,6}$ — see figure. Ironically, the probe, L1.28, that was thought to be revealing a single locus in the 1984 work was demonstrating linkage to both $R P 2$ and $R P 3$, because the DNA sequence it detects, DXS7, is situated between these two loci $^{4,5}$ (see figure).

Second, whereas large chromosomal deletions are generally invaluable guides to disease genes, a large deletion in one RP patient (called B.B.) may have misdirected the search. RP3 mapped close to the B.B. deletion and was initially presumed to be within it. But studies of other RP patients with deletions in the region ${ }^{2}$, and additional mapping ${ }^{9}$, indicated that $R P G R$ is about 400 kilobases centromeric to the nearer edge of the B.B. deletion. In the end, the good reputation of deletions in positional cloning was restored by a

\title{
Retinitis pigmentosa: the Ran connection
}

WHAT biological riches might lie in the RPGR coding sequences that Meindl et $a .^{2}$ have unearthed? The predicted 90K RPGR protein bears significant homology to the RCC1 family of guanine nucleotide exchange factors. RCC1 activates the replacement of guanosine diphosphate (GDP) by guanosine triphosphate (GTP) on the small GTPase, Ran (ref. 13). The RCC1-Ran complex regulates the transport of two different sets of macromolecules through the nuclear pore complex (NPC) ${ }^{14}$. RNA is shuttled out of the nucleus when RCC1, a nuclear protein, activates GTP loading of nuclear Ran. On the cytoplasmic side of the NPC, Ran-GDP first initiates the association of a large complex composed of the NPC, nucleartargeted proteins and a multisubunit transporter. Once this complex is formed, the nuclear-targeted proteins are shuttled through the pore when the
Ran-bound GDP is exchanged for GTP (ref. 15). So Ran-GDP and Ran-GTP each play distinct roles on both sides of the nuclear membrane in mediating the assembly and dissociation of multicomponent complexes.

It seems safe to suggest that the RPGR protein may also regulate nucleotide exchange on Ran, or on a Ranlike protein. Unlike RCC1, however, RPGR contains a long, charged carboxy-terminal domain which ends with a CAAX box, probably targeting this protein for geranylgeranylation and insertion into a cellular membrane. In addition, RPGR does not contain amino-terminal nuclear localization sequences and may therefore reside in some other subcellular compartment. These characteristics of RPGR, and the presence of other novel RCC1-like proteins in the public databases ${ }^{2}$, suggest that Ran proteins may regulate many cellular functions and that different ex- changers will activate GTP-loading of Ran at different cellular locales. Finding out what the RPGR protein does will require identifying its subcellular location, its target GTPase and the macromolecular company it keeps.

The answers to these basic biochemical questions should explain the pathogenesis of this type of XIRP, particularly the unique sensitivity of the retina to mutations in RPGR - an important issue, because the retina and retinal pigment epithelium have the lowest levels of RPGR expression, by far, of all tissues examined ${ }^{2}$. Perhaps the RPGR protein has a preferred retinal partner, such as a Ran-like protein that is not activated by other RCC1 proteins in the retina. In addition, phenotypic questions such as whether ciliary abnormalities occur in XIRP (ref. 16), can now be subjected to molecular scrutiny, and may point to other tissues in which $R P G R$ is essential. S. E. E. \& R. R. Mcl. 